\title{
УЧЁТ ВЛИЯНИЯ ОТНОСИТЕЛЬНЫХ ФАЗОВЫХ ПРОНИЦАЕМОСТЕЙ НА АДАПТАЦИЮ МЕСТОРОЖДЕНИЯ С ТЕРРИГЕННЫМ ТИПОМ КОЛЛЕКТОРА
}

\author{
Коровин Михаил Олегович, \\ korovinmo@hw.tpu.ru \\ Национальный исследовательский Томский политехнический университет, \\ Россия, 634050, г. Томск, пр. Ленина, 30.
}

\begin{abstract}
Актуальность исследования заключается в фракте истощения запасов уелеводородов. Перед исследователями сейчас стоит основная задача - добыть из пластов-коллекторов как можно больше углеводородов. Решить эту проблему можно двумя способами: создать новую технологию формирования цифрровой модели продуктивного резервуара (математическую модель) или новый (альтернативный) подход к обработке существующих данных. В рамках второго способа необходимо провести анализ применения текущих данных, кардинально влияющих на гидродинамические характеристики, и детально изучить возможности их альтернативного учёта в существующих математических моделях, реализованных в современных программных продуктах. Дополнительным опорным фактором является то, что все исследования и результаты контролируются геологическими условиями формирования пластов-коллекторов и их насыщением. Кривые относительных фазовых проницаемостей являются одним из основных наборов данных, критически влияющих на фильтрацию уәлеводородов. Коэфффициент остаточной нефти, воды, точка перехода фрильтрации из зоны преобладания нефрти в зону проебладания воды - важнейшие параметры, от которых в конечном итоге зависит коэффрициент вытеснения.

Цель: изучить возможность использования кривых относительных фазовых проницаемостей для улучшения адаптации характеристик добычи без дополнительных их модификаций и перерасчётов. Предполагается получить дополнительные расчёты гидродинамических моделей и сопоставить эти результаты с фрактическими результатами добычи.

объект: месторождение, территориально расположенное в юго-восточной части Западной Сибири. Тип коллектора терригенный. Стратиграфически пласт приурочен к верхнеюрским отложениям. Неоднородное распределение фрильтрационных свойств позволяет рассмотреть влияние относительных фазовых проницаемостей на расчёты характеристик добычи. Методы: создание гидродинамической модели месторождения с применением неизменённых кривых относительных фразовых проницаемостей. Основная идея заключается в необходимости изучить возможность и целесообразность использования немодиифцированных кривых для расчёта параметров добычи. Финальный шаг - сравнение характеристик добычи по гидроднамическим моделям и фактических данных разработки.

Результаты. Была уточнена фильтрационная модель изучаемого месторождения. Удалось получить наиболее объективную характеристику процесса фильтрации углеводородов на основе уточнённой модели структуры порового пространства. Это очень важно, так как именно структура порового пространства и распределение свойств горных пород являются предопределяющими фракторами, согласно которым будет происходить фильтрация углеводородов. Получаемые лабораторные измерения дают информацию о наиболее приближенном состоянии исходного порового пространства. Установлено, что относительные фразовые проницаемости критически влияют на расчёты накопленной добычи нефрти. Большое количество исследований позволяет охарактеризовать каждую фацию и все фильтрационнье особенности вертикали разреза. Использование исходных кривых относительных фразовых проницаемостей даёт возможность оценить необходимость применения модифрицирования кривых и приблизить математическое описание свойств коллектора к реальной геологической картине.
\end{abstract}

\section{Ключевые слова:}

Анизотропия проницаемости, масштаб анизотропии, гидродинамическое моделирование, относительные фразовые проницаемости, распределение свойств.

\section{Введение}

Относительная фазовая проницаемость - это отношение эффективной проницаемости фазы к абсолютной. Исследований относительной фазовой проницаемости, как правило, очень мало на месторождении. Даже меньше, чем стандартных керновых исследований (пористости, проницаемости). Но они очень важны для понимания характеристик фильтрации и расчётов коэффициента вытеснения нефти. При анализе графиков относительных фазовых проницаемостей определяются точки, на основании которых разграничиваются зоны добычи нефти, нефти и воды, воды и нефти, «100\% воды». Учёные отмечают важные наблюдения и закономерности. Так, например, специалисты [1-4] при сопоставлении окончательных расчётов выявили, что относительные фазовые проницаемости, применяемые в моделях и в результате оценки данных добычи, существенно разнятся. С увеличением доли обводнения продукции ожидается повышение степени сопротивления фильтрации в процессе вытеснения водой нефти. Авторы отмечают, что такого эффекта не отмечается. Дополнительно авторы указывают на завышенные расчётные значения коэффициента вытеснения, которые не подтверждаются разработкой и, более того, оказываются заниженными по факту. Это является существенной проблемой, так как необходимо точно знать сколько углеводородов можно добыть при текущих параметрах модели.

\section{Общепринятые подходы к применению} данных фазовых проницаемостей

Основные подходы, которыми пользуются при создании геологических и гидродинамических моделей, изложены в работах $[1,5-13]$ и включают: осредне- 
ние кривых относительных фазовых проницаемостей; масштабирование значений по оси абсцисс; масштабирование значений по осям абсцисс и ординат.

Это делается для адаптации фактических данных добычи и расчётных параметров по гидродинамической модели. Основной недостаток всех этих подходов - в модификации данных. Каждый из них предполагает проведение дополнительных расчётов и изменение расчётных данных. Эти подходы направлены на то, чтобы создать одну единственную модель относительных фазовых проницаемостей (ОФП) на пласт или на всё месторождение. Такие подходы оправданы небольшим количеством исследований и ещё одним важным моментом. Исследования, как правило, разрознены, и разграничить зоны влияния кривых относительных фазовых проницаемостей на части пласта или по площади месторождения не представляется возможным.

Таким образом, есть три стандартных подхода при обработке и создании единой модели вытеснения с помощью относительных фазовых проницаемостей. Все они направлены на создание единых кривых: относительной фазовой проницаемости по воде и по нефти для всего пласта или для всего месторождения. Эти методики отработаны и стандартизованы в исполнении и применении. Но отдельной проблемой становится арифметическая модификация и масштабирование кривых, что приводит к потере отдельных деталей, характеризующих вытеснение, и усредняет все кривые. В этом случае исчезают и нивелируются особенности гидродинамических процессов конкретной области, из которой были взяты образцы керна и проведены исследования относительных фазовых проницаемостей.

\section{Альтернативный подход к имплементации данных фазовых проницаемостей}

В данной работе предлагается рассмотреть использование в каждой области месторождения своих кривых относительных фазовых проницаемостей. Это приведёт к наличию латеральных зон на месторождении, каждая из которых будет характеризоваться своими кривыми относительных фазовых проницаемостей, и, соответственно, гидродинамическая картина вытеснения флюидов будет различаться. Стандартные подходы также будут обязательно рассмотрены для сравнения результатов с расчётами по предлагаемому подходу.

Цель работы - изучить возможность использования кривых относительных фазовых проницаемостей без их модификаций и перерасчётов. Предполагается получить дополнительные расчёты гидродинамических моделей и сопоставить рассчитанные результаты с фактическими результатами добычи.

Научная новизна данного исследования заключается в представлениях о зональном характере вытеснения нефти, учитывая, что особенности вытеснения напрямую связаны с обстановкой осадконакопления. Это позволяет в зонах с одинаковыми фациальными маркерами использовать одинаковый набор кривых относительных фазовых проницаемостей.
Таким образом, снимается вопрос об усреднении и масштабировании. Эти операции связаны с дополнительными пересчётами, потерей детальности данных и необходимостью обоснования выбора уравнений для масштабирования. Однако возникает другая сложность, которая заключается в достоверном выделении границ фациальных зон. Полученный результат, основанный на сопоставлении расчётных и фактических данных добычи, позволит проанализировать поведение флюидов в пласте с исходными характеристиками вытеснения

Специалисты отмечают, что операции масштабирования, которой подвергаются кривые относительных фазовых проницаемостей, значительно влияют на расчёты характеристик добычи углеводородов.

На этом этапе хотелось бы заострить внимание ещё на одном важном моменте. Это факторы, которые оказывают влияние на относительные фазовые проницаемости. Среди них непосредственно поровое пространство (структура, смачиваемость), свойства пластовых флюидов (физические и химические), направление исходной фильтрации углеводородов, скорость флюидофильтрации, температура [6]. Таким образом, характер исходных кривых относительных фазовых проницаемостей будет отражать все эти особенности при анализе данных кривых. В случае их изменения при формировании модифицированных ОФП будет происходить искажение картины фильтрации, что приведёт к расхождению коэффициента вытеснения, вычисленного и полученного по материалам анализа разработки. Геологам и разработчикам нефтяных и газовых месторождений необходимо учитывать важность исходных данных и то, что любые изменения и модификации неизбежно отразятся на увеличении погрешности (в лучшем случае), а возможно, и приведут к дополнительным действиям по адаптации добычи в будущем (пессимистичный вариант развития событий по адаптации расчётов модели и фактической истории разработки) [7-13].

На рис. 1 показан наглядный пример результатов лабораторных исследования (кривые Oil, Water) и данных, подверженных модификацией (Oil_m, Water_m) по рассматриваемому в статье месторождению, где отчётливо видно, что точка пересечения модифицированных кривых смещена вправо. Это предполагает улучшенные характеристиках пласта по усреднённым кривым. По модифицированным кривым точка пересечения находится на уровне 0,78 д.е. водонасыщенности, что свидетельствует о наличии переходной зоны на этой точке и изменении фильтрации с нефти на воду при увеличении водонасыщенности. По кривым отдельного исследования (Oil, Water) точка пересечения находится на уровне 0,57 д.е. Получается, что модифицированные кривые завышают точку пересечения кривых и показывают более оптимистичный вариант представления распределения нефти и воды. Становится очевидным, что данный параметр критически влияет на характеристики добычи углеводородов и фильтрации жидкости при добыче. 


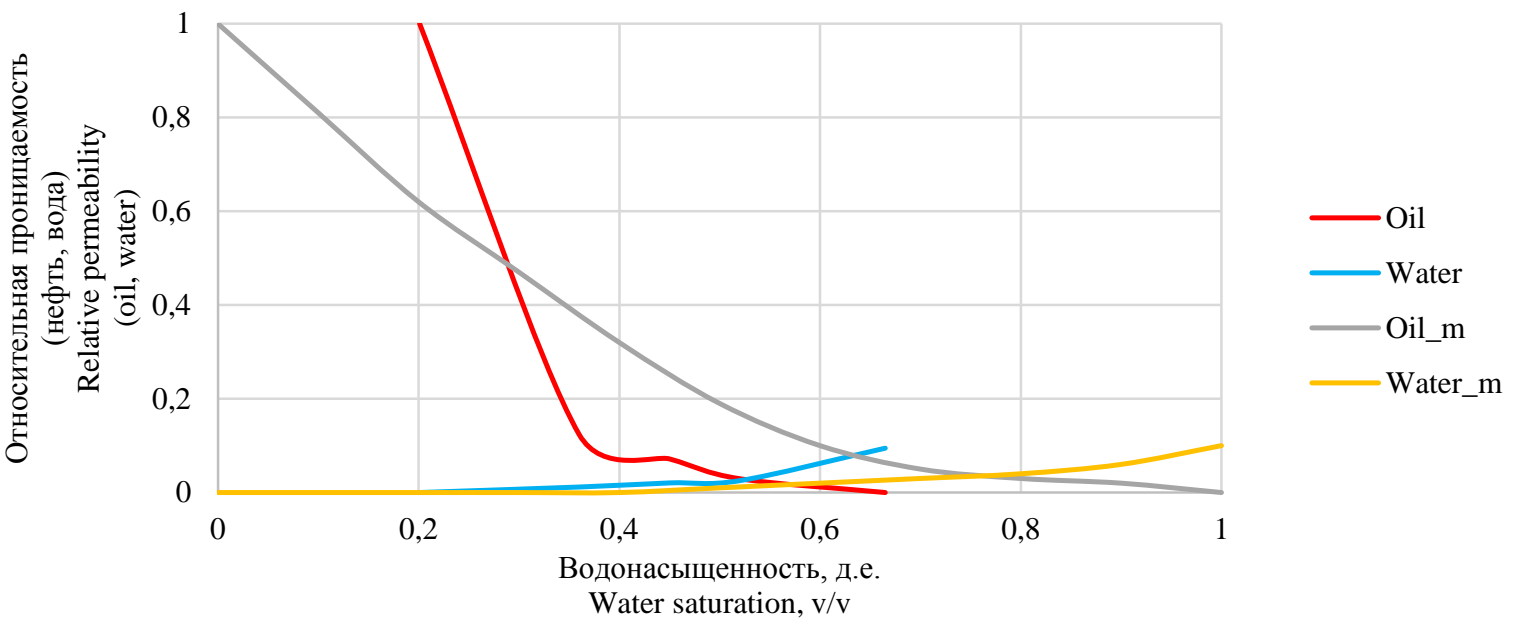

Рис. 1. Реальные и модифицированные относительные фазовые проницаемости по нефти и воде анализируемого месторождения

Fig. 1. Relative phase permeabilities of oil and water for research field

Схема представления фильтрации жидкости осложняется ещё и тем, что в модифицированных кривых присутствуют области значений, которых нет в исходных данных. Это значения проницаемостей при значениях водонасыщенности менее 0,201 и более 0,665 д.е. При использовании исходных кривых фильтрация «чистой нефти» будет происходить в области водонасыщенности менее 0,201 , а фильтрация «100 \% воды» при значениях водонасыщенности более 0,665 д.е. При использовании модифицированных кривых эти значения составляют 0,4 и 1 соответственно. Получается, что в зоне проведения исходных исследований при стандартном подходе создания геологической модели кривые смещены в зону завышенного нефтенасыщения, а это впоследствии приводит к завышенной оценке коэффициента вытеснения.

Цель проводимых расчётов и исследований заключается в попытке избавиться от необходимости модификации ОФП, что позволяет проанализировать расчёты гидродинамической модели и выяснить, возможно ли обойтись без операции усреднения. При этом будут контролироваться параметры гидродинамической модели и детальные расчёты добычи. Дополнительно необходимо обратить внимание на площадь влияния каждого отдельного испытания относительных фазовых проницаемостей. В случае модифицированных кривых один полученный набор используется для всего месторождения. В рассматриваемой модели коллектора возникает необходимость каким-то образом создать переходы между разными зонами влияния того или иного набора кривых относительных фазовых проницаемостей. Предлагается следующая последовательность действий, учитывающая плотность исследований, распределение фациальных обстановок осадконакопления, ограниченность площади непроницаемыми тектоническими нарушениями. Зоны распространения исходных кривых ограничиваются сменой обстановки осадконакопления или наличием скважины с исследованиями относительных фазовых проницаемостей (в этом случае зона влияния ограничивается сере- диной расстояния между скважинами). Исследования относительных фазовых проницаемостей и миниграфики с фазовыми проницаемостями отражены на рис. 2. Легенда аналогична рис. 1 с тем важным моментом, что отражены исходные фазовые проницаемости без масштабирования. Анализируемое месторождение детально охарактеризовано с точки зрения геологического строения [14-16]. Пробуренные скважины закрашены синим цветом, а скважины с исследованиями относительных фазовых проницаемостей закрашены красным цветом. Таких скважин всего 11, но здесь необходимо отметить, что они хорошо распределены по площади - каждая из частей месторождения охвачена исследованиями. Основные площади обстановок осадконакопления тоже получаются охваченными, за исключением гребней барьерных островов и задернованных частей барьерных островов. Данные распределены таким образом, что в каждой области месторождения (северной, центральной, южной) существуют собственные исследования относительных фазовых проницаемостей, которые и будут использоваться для создания гидродинамической модели.

Дополнительно необходимо упомянуть, что единый усреднённый набор кривых фазовых проницаемостей ведёт к дополнительным операциям по адаптации месторождения. Смещая точку пересечения фазовых проницаемостей, необходимо помнить, что это непосредственно влияет на фильтрацию флюидов и оценку скорости добычи запасов [17-19]. При использовании исходных кривых специалисты избавляются от контроля смещения точки и проверки правильности проведённых расчётов по модификации кривых. Единственный вопрос остаётся в определении зоны влияния конкретных исследований. Для его решения необходимо использовать данные фациальных исследований, которые наглядно показывают распределение компонент фациальных составляющих обстановок осадконакопления. А это, в свою очередь, несёт информацию об изменении режима и направления фильтрации [20-25]. 


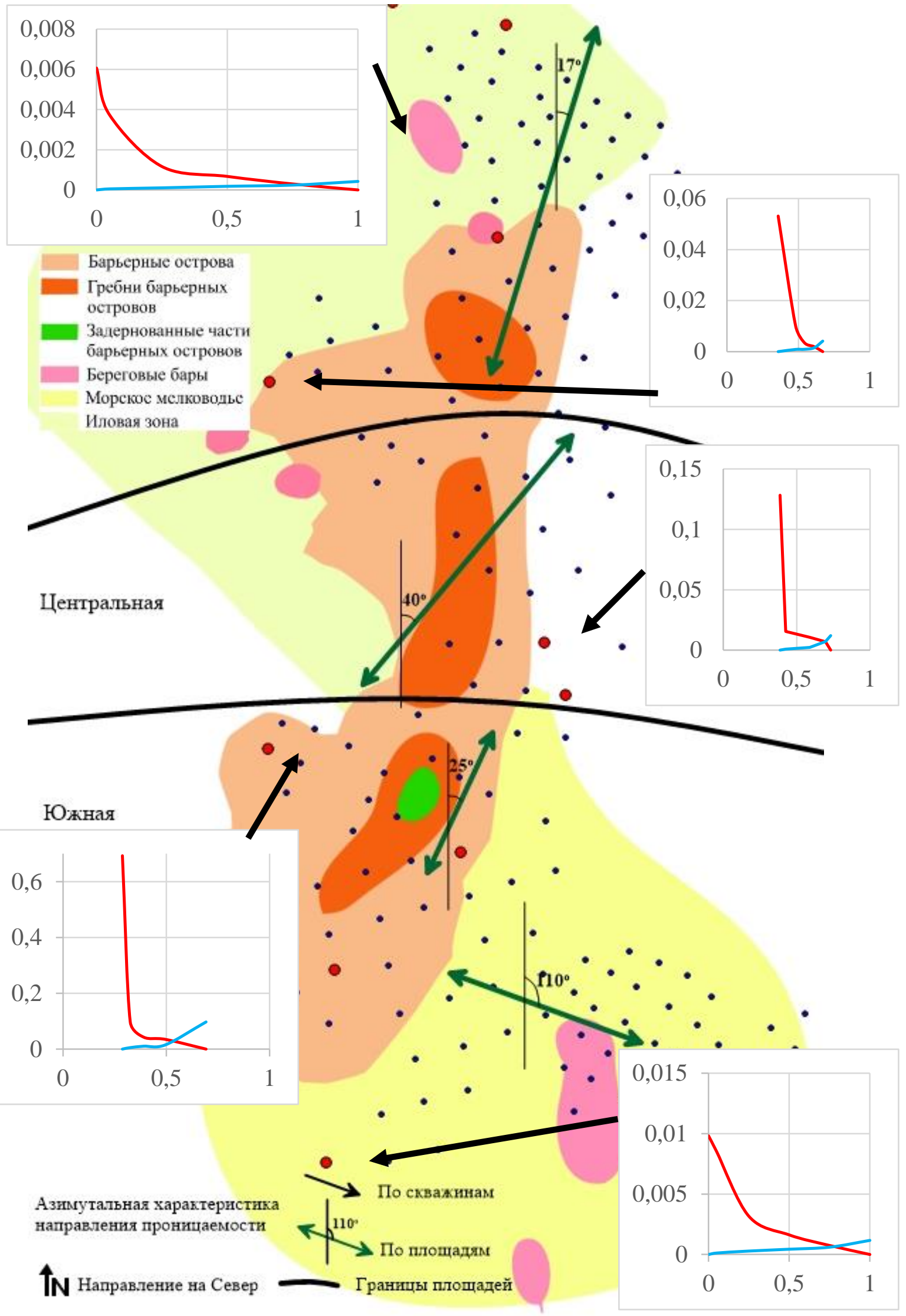

Рис. 2. Распределение фациальных обстановок. Скважсины (синие), ОФП (красные)

Fig. 2. Facies distribution. Wells (blue), relative permeability (red) 
Накопленная добыча нефти - параметр, по которому оценивается качество адаптации созданной гидродинамической модели. На рис. 3 показаны графики накопленной добычи нефти по всему месторождению. Расположение графиков показывает более близкое соотношение накопленной добычи, вычисленной по модели с исходными кривыми относительных фазовых проницаемостей. Модели по модифицированным кривым были рассчитаны и получены для всей площади месторождения.

Полученные результаты показывают важность учёта двух факторов:

- отсутствие модификаций кривых относительных фазовых проницаемостей;

- определение фациальных обстановок осадконакопления и сопоставления определённых обстановок с наборами кривых относительных фазовых проницаемостей.

Обстановка осадконакопления предопределяет (является одним из важнейших факторов) гидродинамические характеристики пласта-коллектора. Поэтому при отсутствии исследований кривых относительных фазовых проницаемостей в некоторых частях месторождения такая проблема решается путём использования набора кривых с блока месторождения со схожей обстановкой осадконакопления и схожими свойствами коллектора и флюидов.

По расчётам получается, что накопленная добыча нефти становится ближе к фактической при использовании исходных кривых относительных фазовых проницаемостей по нефти и воде без усреднений и модификаций. Такие расчёты позволяют изменить подход к созданию гидродинамической модели. С одной стороны, специалисты избавляются от необходимости дополнительно пересчитывать кривые относительных фазовых проницаемостей. С другой стороны, необходимо большее внимание уделить распределению фациальных обстановок и созданию зон разграничения влияния каждого набора кривых относительных фазовых проницаемостей. Геологически и математически такой подход будет более приближен к получению с помощью гидродинамической модели добычи нефти наиболее точных данных. Геологически подход сводится к более детальному учёту каждого исследования кривых относительных фазовых проницаемостей и сопоставлению с фациальными категориями обстановок осадконакопления. Уменьшается количество математических операций, что ведёт к снижению погрешностей и неопределённости.

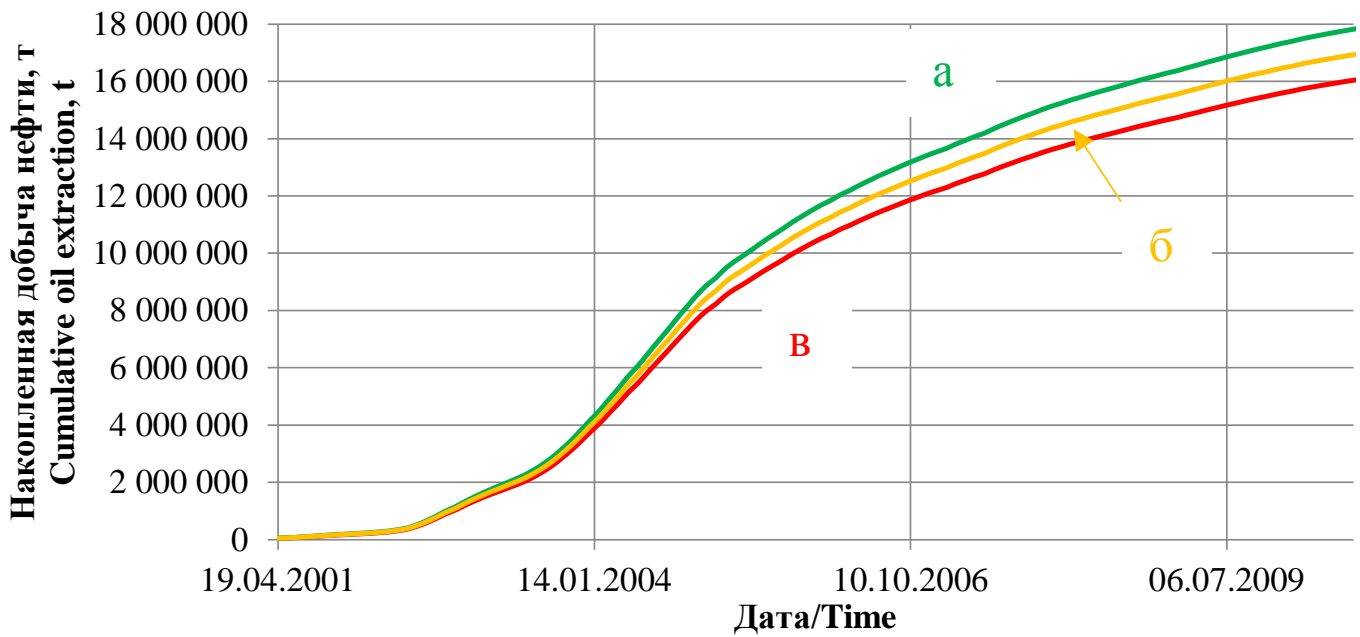

Рис. 3. Графики накопленной добычи нефти в процессе разработки залежи (а), рассчитанной по модели с исходными (б) и модифицированными (в) кривыми ОФП

Fig. 3. Cumulative oil extraction comparison during the development (a), calculated with initial (b), modified (c) relative permeability curves

\section{Заключение и выводы}

Осреднение кривых относительных фазовых проницаемостей значительно влияет на итоговые расчёты добычи по гидродинамической модели.

Варианты гидродинамических моделей без осреднения показывают более приближенное состояние добычи к фактическим данным. Дополнительным осложняющим фактором служит выделение границ влияния каждого отдельного набора кривых относительных фазовых проницаемостей. Для решения этой проблемы предлагаются следу- ющие подходы: применение карты распределения фациальных тел; прослеживание границ литологических составов пород по геофизическим исследованиям скважин; отметка границы влияния фации на среднем расстоянии между скважинами с исследованиями.

По степени точности получаемых результатов они расположены снизу вверх (чем выше, тем точнее). Применение карты распределения фациальных тел предоставит наилучший результат учёта влияния фазовых проницаемостей по площади. 


\section{СПИСОК ЛИТЕРАТУРЬ}

1. Захаров Я.В., Шайхутдинов Д.К. Масштабирование относительных фазовых проницаемостей как шаг к повышению качества гидродинамического моделирования // Территория «НЕФТЕГАЗ». - 2015. - № 10. - С. 88-93.

2. Методы определения функций относительной фазовой проницаемости в задачах многофазной фильтрации / А.Б. Шабаров, А.В. Шаталов, П.В. Марков, Н.В. Шаталова // Вестник Тюменского государственного университета. Физикоматематическое моделирование. Нефть, газ, энергетика. 2018. - T. 4. - № 1. - C. 79-109. DOI: $10.21684 / 2411-7978-$ 2018-4-1-79-109.

3. Родионов С.П., Соколюк Л.Н. Расчёт и использование модифицированных относительных фазовых проницаемостей при преобразовании геологической модели в гидродинамическую // Прикладная механика, динамика жидкости и газа ТРУДЫ МФТИ. - 2010. - Т. 2 - № 2 - С. 130-136.

4. Insights, trends and challenges associated with measuring coal relative permeability / D. Shaw, P. Mostaghimi, F. Hussain, R.T. Armstrong // E3S Web of Conferences. - 2019. - DOI: doi.org/10.1051/e3sconf/20198901004

5. Li K., Horne R.N. Comparison of methods to calculate relative permeability from capillary pressure in consolidated water-wet porous media // Water Recourses. - 2006. - V. 42. - W06405. DOI: 10.1029/2005WR004482

6. Колесник С.В., Трофимов А.С., Полищук С.Т. Относительная фазовая проницаемость. - Тюмень: ТюмГНГУ, 2013. - 96 с.

7. Сотников О.С. Совершенствование методов определения относительных фазовых проницаемостей и их применения при гидродинамическом моделировании разработки нефтяных месторождений: дис. ... канд. техн. наук. - Бугульма, 2009. $155 \mathrm{c}$

8. Фатихов С.3., Сыртланов В.Р. К вопросу вычисления относительных фазовых проницаемостей // Электронный научный журнал «Нефтегазовое дело». - 2012. - № 1. - С. 324-334.

9. Validation of permeability and relative permeability data using mercury injection capillary pressure data / R. Arabjamaloei, D. Daniels, E. Ebeltoft, E. Petersen, R.J. Pitman, D. Ruth // E3S Web of Conferences. - 2019. DOI: doi.org/10.1051/e3sconf/20198901001

10. Experimental estimation of relative permeabilities through computed tomography / A.F.O. Meneses, M. Carrillo, L.F. Otero, E.H. Herrera, N. Santos // SPWLA 60th Annual Logging Symposium. - 2019. DOI: doi.org/10.30632/T60ALS-2019_NNNN

11. Дмитриев Н.М., Максимов В.М. О структуре тензоров коэффициентов фазовых и относительных проницаемостей для анизотропных пористых сред // Докл. РАН. - 1998. - Т. 358. № 3. - C. 337-339.

12. Noaman El-K. The application of Buckley-Leverett displacement to waterflooding in non-communicating stratified reservoirs // Proceedings of the Middle East Oil Show. - 2001. DOI: 10.2118/68076-MS.

13. Influence of capillarity onrelative permeability in fractional flows / S. Zou, Y. Liu, J. Cai, R.T. Armstrong // Water Resources Research. - 2020. DOI: doi.org/10.1029/2020WR027624

14. Конторович В.А. Тектоника и нефтегазоносность мезозойскокайнозойских отложений юго-восточных районов Западной Сибири. - Новосибирск: Изд-во СО РАН, 2002. - 253 с.
15. Роль мезозойско-кайнозойской тектоники в формировании залежей углеводородов в южных частях Каймысовского свода и Нюрольской мегавпадины / В.А. Конторович, М.В. Соловьев, Л.М. Калинина, А.Ю. Калинин // Геология и геофизика. 2011. - T. 8. - C. 1075-1091.

16. Коровин М.О., Меркулов В.П. Направления фильтрации флюидов на месторождениях нефти юго-восточной части Каймысовского свода // Газовая промышленность научнотехнический и производственный журнал. - 2014. - Спец. вып. (№ 708). - С. 90-94.

17. Использование метода нестационарной фильтрации для оценки влияния скорости фильтрации на относительные фазовые проницаемости / Д.М. Орлов, А.Л. Федосеев, Н.В. Савченко, И.Ю. Корчажкина, Б.А. Григорьев, А.Е. Рыжов, Т.А. Перунова, Н.Ю. Максимова, Е.П. Калашникова // Научно-технический сборник. Вести газовой науки Проблемы разработки и эксплуатации газовых, газоконденсатных и нефтегазоконоденсатных месторождений - 2015. - № 3 (23). - С. 8-14.

18. Тудвачев А.В., Коносавский П.К. Анализ и прогнозирование зависимостей функции фазовой проницаемости нефтенасыщенных коллекторов на примере месторождений Сургутского и Вартовского сводов Западно-Сибирской нефтегазоносной провинции // Вестник СпбГУ. - 2013. - Cер. 7. - Вып. 1. C. $31-41$.

19. Белошапка И.Е., Ганиев Д.И. Применение фильтрационных исследований для изучения технологий разработки месторождений нетрадиционных коллекторов и трудноизвлекаемых запасов нефти // Вестник Российского университета дружбы народов. Серия: Инженерные исследования. - 2018. - Т. 19. № 3. - C. 343-357. DOI: 10.22363/2312-8143-2018-19-3-343-357.

20. Пономарёв А.И. Повышение эффективности разработки залежей углеводородов в низкопроницаемых и слоистонеоднородных колекторах: дис. ... д-ра техн. наук. - Уфа, 2000. $-457 \mathrm{c}$

21. Кузнецов А.М. Научно-методические основы исследования влияния свойств пород-коллекторов на эффективность извлечения углеводородов из недр: дис. д-ра техн. наук. - М., 1998. $-280 \mathrm{c}$

22. Ушаков В.В. Определение технологических показателей разработки при вытеснении парафинистой нефти водой: дис. ... канд. техн. наук. - М., 1984. - 168 c.

23. Quantification of uncertainty and best practice in computing interfacial curvature from complex pore space images / T. Akai, Q.-Lin, A. Alhosani, B. Bijeljic, M.J. Blunt // Materials. - 2019. V. 12 (13). DOI: doi.org/10.3390/ma12132138

24. Dynamic capillary pressure analysis of tight sandstone based on digital rock model / Y. Cao, M. Tang, Q. Zhang, J. Tang, S. Lu // Capillarity. 2020. - V. 3 (2). DOI: doi.org/10.46690/capi.2020.02.02

25. Zou S., Armstrong R. Multiphase flow under heterogeneous wettability conditions studied by special core analysis and pore scale imaging // SPE Journal. - 2019. - V. 24 (3). DOI: doi.org/10.2118/195577-PA

Поступила 10.03.2021 2.

\section{Информация об авторах}

Коровин М.O., кандидат геолого-минералогических наук, доцент отделения нефтегазового дела Инженерной школы природных ресурсов Национального исследовательского Томского политехнического университета. 
UDC 550.3

\title{
RELATIVE PERMEABILITY IMPLEMENTATION INSIDE TERRIGENEOUS OIL FIELD RESERVOIR
}

\author{
Mikhail O. Korovin, \\ korovinmo@hw.tpu.ru \\ National Research Tomsk Polytechnic University, \\ 30, Lenin avenue, Tomsk, 634050, Russia.
}

\begin{abstract}
The relevance of the research lies in an important fact related to the depletion of hydrocarbon reserves. The main task for researchers now is to extract as much hydrocarbons as possible from the reservoirs. There are two ways to solve this problem: create a new technology (mathematical model) or a new (alternative) approach to processing existing data. It is necessary to analyze the application of current data, which radically affect the hydrodynamic characteristics and to study in detail the possibilities of their alternative accounting in existing mathematical models implemented in modern software products. An additional support factor is that all studies and results are controlled by the geological conditions of reservoir formation and their saturation. Relative phase permeability curves are one of the main datasets that critically affect hydrocarbon filtration. The coefficient of residual oil, water, the point of transition of filtration from the zone of predominance of oil to the zone of penetration of water are the most important parameters on which the displacement coefficient ultimately depends.

The aim of this work is to study the possibility of using curves of relative phase permeabilities without additional modifications and recalculations. It is expected to obtain additional simulations of hydrodynamic models and compare these results with actual production results.

The study object is a field geographically located in Western Siberia. Reservoir type is terrigenous. Stratigraphically, the stratum is confined to the Upper Jurassic deposits. The inhomogeneous distribution of filtration properties allows us to consider the effect of relative phase permeabilities on the calculation of production characteristics.

Methods: creation of a hydrodynamic model of the field using unchanged curves of relative phase permeabilities. The main idea is to study the possibility and feasibility of using unmodified curves for calculating production parameters. The final step is a comparison of production characteristics from hydrodynamic models and actual development data.

Results. The filtration model of the studied field was refined. It was possible to get the most accurate idea of the filtration of hydrocarbons. It becomes possible to take into account the structure of the pore space more detailed than previously. This is very important, since it is the structure of the threshold space and the distribution of the properties of rocks that are the predetermining factors according to which the filtration of hydrocarbons will take place. The resulting laboratory measurements provide information about the state that is closest to the original pore space. It was found that the relative permeabilities of the phase critically affect the calculation of cumulative oil production. $A$ large number of studies make it possible to characterize each facies and all filtration features of the vertical section. The use of the initial curves of relative phase permeabilities makes it possible to assess the need to apply modification of the curves and to bring the mathematical description of the reservoir properties closer to the real geological picture.
\end{abstract}

\section{Key words:}

Permeability anisotropy, anisotropy scale, simulation modeling, relative permeabilities, properties distribution.

\section{REFERENCES}

1. Zakharov Ya.V., Shaykhutdinov D.K. Masshtabirovanie otnositelnykh fazovykh pronitsaemostey kak shag k povysheniyu kachestva gidrodinamicheskogo modelirovaniya [Upscaling of relative phases permeabilities improves reservoir modeling]. Territoriya «NEFTEGAZ», 2015, vol. 10, pp. 88-93.

2. Shabarov A.B., Shatalov A.V., Markov P.V., Shatalova N.V Metody opredeleniya funktsiy otnositelnoy fazovoy pronitsaemosti $\mathrm{v}$ zadachakh mnogofaznoy filtratsii [Methods for determining the functions of relative phase permeability in problems of multiphase filtration]. Vestnik Tjumenskogo gosudarstvennogo universiteta. Fiziko-matematicheskoe modelirovanie. Neft, gaz, energetika, 2018, vol. 4, no. 1, pp. 79-109. DOI: 10.21684/2411-7978-20184-1-79-109.

3. Rodionov S.P., Sokolyuk L.N. Raschet i ispolzovanie modifitsirovannykh otnositelnykh fazovykh pronitsaemostey pri preobrazovanii geologicheskoy modeli v gidrodinamicheskuyu [Calculation and use of modified relative phase permeabilities when converting a geological model into a hydrodynamic one]. Prikladnaya mekhanika, dinamika zhidkosti i gaza. TRUDY MFTI, 2010, vol. 2, no. 2 , pp. 130-136.

4. Shaw D., Mostaghimi P., Hussain F., Armstrong R.T. Insights, trends and challenges associated with measuring coal relative permeability. E3S Web of Conferences, 2019. DOI: doi.org/10.1051/e3sconf/20198901004

5. Li K., Horne R.N. Comparison of methods to calculate relative permeability from capillary pressure in consolidated water-wet porous media. Water Recourses, 2006, vol. 42. DOI: https://10.1029/2005WR004482.
6. Kolesnik S.V., Trofimov A.S., Polishchuk S.T. Otnositelnaya fazovaya pronitsaemost [Relative phase permeability]. Tyumen, TyumGNGU Publ., 2013. 96 p

7. Sotnikov O.S. Sovershenstvovanie metodov opredeleniya otnositelnykh fazovykh pronitsaemostey $i$ ikh primeneniya pri gidrodinamicheskom modelirovanii razrabotki neftyanykh mestorozhdeniy. Dis. Kand. nauk [Improvement of methods for determining relative phase permeabilities and their application in hydrodynamic modeling of oil field development. Cand. Diss.]. Bugulma, 2009, p. 155.

8. Fatikhov S.Z., Syrtlanov V.R. K voprosu vychisleniya otnositelnykh fazovykh pronitsaemostey [On the issue of calculating relative phase permeabilities]. Elektronny nauchny zhurnal «Neftegazovoe delo», 2012, no. 1, pp. 324-334.

9. Arabjamaloei R., Daniels D., Ebeltoft E., Petersen E., Pitman R.J., Ruth D. Validation of Permeability and Relative Permeability Data Using Mercury Injection Capillary Pressure Data. E3S Web of Conferences, 2019. DOI: https://doi.org/10.1051/e3sconf/ 20198901001

10. A.F.O. Meneses, Carrillo M., L.F. Otero, Hernando Herrera E., Santos N. Experimental estimation of relative permeabilities through computed tomography. SPWLA $60^{\text {th }}$ Annual Logging Symposium. 2019. DOI: https://doi.org/10.30632/T60ALS2019_NNNN

11. Dmitriev N.M., Maksimov V.M. O strukture tenzorov koeffitsientov fazovykh i otnositelnykh pronitsaemostey dlya anizotropnykh poristykh sred [On the structure of tensors of coefficients of phase and relative permeabilities for anisotropic porous media]. Dokl. RAN, 1998, vol. 358, no. 3, pp. 337-339. 
12. Noaman El-K. The application of Buckley-Leverett displacement to waterflooding in non-communicating stratified reservoirs. Proceedings of the Middle East Oil Show, 2001. DOI: 10.2118/68076MS.

13. Zou S., Liu Y., Cai J., Armstrong R.T. Influence of capillarity on relative permeability in fractional flows. Water Resources Research, 2020. DOI: https://doi.org/10.1029/2020WR027624

14. Kontorovich V.A. Tektonika i neftegazonosnost mezozoyskokaynozoyskikh otlozheniy yugo-vostochnykh rayonov Zapadnoy Sibiri [Tectonics and oil-and-gas bearing of the MesozoicCenozoic deposits in southeastern of the Western Siberia]. Novosibirsk, SO RAN Publ., 2002. 253 p.

15. Kontorovich V.A. Solovev M.V., Kalinina L.M., Kalinin A.Yu. The role of the Mesozoic-Cenozoic tectonics in the formation of hydrocarbon deposits in the southern parts of the Kaimysov arch and the Nyurol megadepression. Geology and Geophysics, 2011, no. 8, pp. 1075-1091. In Rus.

16. Korovin M.O., Merkulov V.P. Directions of fluid filtration in oil fields of the southeastern part of the Kaimysovsky arch. Gazovaya promyshlennost, 2014, Special Iss. (no. 708), pp. 90-94. In Rus.

17. Orlov D.M., Fedoseev A.L., Savchenko N.V., Korchazhkina I.Yu., Grigorev B.A., Ryzhov A.E., Perunova T.A., Maksimova N.Yu. Kalashnikova E.P. Ispolzovanie metoda nestatsionarnoy filtratsii dlya otsenki vliyaniya skorosti filtratsii na otnositelnye fazovye pronitsaemosti [Using the non-stationary filtration method to assess the effect of filtration rate on relative phase permeabilities] Nauchno-tekhnicheskiy sbornik. Vesti gazovoy nauki. Problemy razrabotki i ekspluatatsii gazovykh, gazokondensatnykh i neftegazokonodensatnykh mestorozhdeniy, 2015, vol. 3 (23), pp. 8-14.

18. Tudvachev A.V., Konosavskiy P.K. Analysis and prediction of the dependences of the phase permeability function of oil-saturated reservoirs on the example of the fields of the Surgut and Vartovsky arches of the West Siberian oil and gas province. Vestnik SpbGU, 2013, Ser. 7, vol. 1, pp. 31-41. In Rus.

19. Beloshapka I.E., Ganiev D.I. Application of filtration studies to study technologies for the development of unconventional reser- voirs and hard-to-recover oil reserves. Vestnik Rossiyskogo universiteta druzhby narodov. Seriya: Inzhenernye issledovaniya, 2018, vol. 19, no. 3, pp. 343-357. In Rus. DOI 10.22363/2312-81432018-19-3-343-357.

20. Ponomarev A.I. Povyshenie effektivnosti razrabotki zalezhey uglevodorodov $v$ nizkopronicaemykh $i$ sloisto-neodnorodnykh kolektorakh. Dis. Dokt. nauk [Increasing the efficiency of developing hydrocarbon deposits in low-permeability and layered heterogeneous reservoirs. Dr. Diss.]. Ufa, 2000. 457 p.

21. Kuznetsov A.M. Nauchno-metodicheskie osnovy issledovaniya vliyaniya svoystv porod-kollektorov na effektivnost izvlecheniya uglevodorodov iz nedr. Dis. Dokt. nauk [Scientific and methodological foundations for studying the effect of reservoir rock properties on the efficiency of hydrocarbon extraction from the subsoil. Dr. Diss.]. Moskcow, 1998. 280 p.

22. Ushakov V.V. Opredelenie tekhnologicheskikh pokazateley razrabotki pri vytesnenii parafinistoy nefti vodoy. Dis. Kand. nauk [Determination of technological indicators of development during the displacement of paraffinic oil by water. Cand. Diss.]. Moskcow, 1984. $168 \mathrm{p}$

23. Akai T., Lin Q., Alhosani A., Bijeljic B., Blunt M.J. Quantification of uncertainty and best practice in computing interfacial curvature from complex pore space images. Materials, 2019, vol. 12 (13). DOI: https://doi.org/10.3390/ma12132138

24. Cao Y., Tang M., Zhang Q., Tang J., Lu S. Dynamic capillary pressure analysis of tight sandstone based on digital rock model. Capillarity, 2020, vol. $3 \quad$ (2). DOI: https://doi.org/10.46690/capi.2020.02.02

25. Zou S., Armstrong R. Multiphase flow under heterogeneous wettability conditions studied by special core analysis and pore scale imaging. SPE Journal, 2019, vol. 24 (3). DOI: https://doi.org/10.2118/195577-PA

Received: 10 March 2021.

\section{Information about the authors}

Mikhail O. Korovin, Cand. Sc., associate professor, National Research Tomsk Polytechnic University. 